\title{
On integrable rational potentials of the Dirac equation
}

\author{
Tomasz Stachowiak* \\ Center for Theoretical Physics PAS, \\ Al. Lotnikow 32/46, 02-668 Warszawa, Poland \\ Maria Przybylska \\ Institute of Physics, University of Zielona Góra, \\ Licealna 9, 65-417 Zielona Góra, Poland
}

\begin{abstract}
The one-dimensional Dirac equation with a rational potential is reducible to an ordinary differential equation with a Riccati-like coefficient. Its integrability can be studied with the help of differential Galois theory, although the results have to be stated with recursive relations, because in general the equation is of Heun type. The inverse problem of finding integrable rational potentials based on the properties of the singular points is also presented; in particular, a general class of integrable potentials leading to the Whittaker equation is found.
\end{abstract}

\section{INTRODUCTION}

The goal of this work is to examine the solvability of the Dirac equation, when reduced to one spatial dimension. The key point is of course that the analysis is easier and deeper for an ordinary differential equation, and also that the reduction itself can be achieved by many means, such as separating the radial from the angular part, or neglecting two directions in the Cartesian coordinates. A natural source of the potential is the magnetic field, which also provides a direct connection with systems of quantum optics and a possible experimental realization [1]. The resulting equation form is also encountered when dealing with the supersymmetric Schrödinger equation [2], to which all results on integrability can be transferred. Of course, one has to keep track of the different definitions of potentials and physical interpretation, but the mathematical structure remains the same, and that is the main interest of the present Letter.

A great many of known solutions were obtained for the scale invariant potentials, and can be found in [3]. However, such approach relies on the assumption of a particular symmetry and is not exhaustive. Not disregarding it, it seems natural to also consider equations like this (i.e. second order and linear) on grounds of the differential Galois group theory. Mostly because it provides rigorous tools for finding the exact forms of solutions and also because it strictly rules out unsolvable cases - exactly like the "ordinary" Galois theory for polynomials.

Since linear equations often present themselves as central, like the Schrödinger equation or the density perturbation in cosmology, they have already been successfully studied with these methods, e.g. in [2, 4 6 ]. When it comes to the Dirac equation, the complete analysis in the case of general polynomial potentials was carried out in a previous work by one of the authors [7]. This class happened to be very restrictive, and only one non-trivial case - the so-called Dirac oscillator - is explicitly integrable.

The next obvious step seems to be the wider class of rational functions which is undertaken here. This turns out to be too wide to be treated as completely as polynomials, the basic reason being that it is the poles of the equation's coefficients that matter, and polynomials have just one - at infinity. As will be shown, even the simplest potential with just one pole and linear polynomial part cannot be analyzed explicitly in that it requires recursion to formulate integrability conditions. This is a typical feature of the Heun equation and its generalizations, and thus a generic rational potential is beyond explicit classification which was possible for polynomial ones. Fortunately, the recursive conditions can be studied to some extent with regard to the real values of the parameters, and shed some light on the number of admissible physical cases.

The analogy with polynomials also motivates the study of potentials which give rise to similar behavior of the equation's singular points and since direct analysis is impossible, one might also try the inverse problem. Thus, additionally, two other scenarios are considered here: rational potentials which produce only the irregular singularity at infinity, and the Whittaker equation, whose solvability is known and can be used to obtain a new, two-parameter family of integrable potentials.

\footnotetext{
*Electronic address: stachowiak@cft.edu.pl

${ }^{\dagger}$ Electronic address: M.Przybylska@proton.if.uz.zgora.pl
} 


\section{SKETCH OF THE GALOIS THEORY}

To be more specific, let us consider the same setup as in [7], so that the time-independent Dirac equation in one dimension $z$ is

$$
E \psi=\left(-i \boldsymbol{\alpha}_{3} \partial_{z}+\boldsymbol{\beta} m\right) \psi
$$

which gives the second order equations for each spinorial component

$$
\psi_{1,2}^{\prime \prime}(z)=\left(V(z)^{2} \pm V^{\prime}(z)-E^{2}\right) \psi_{1,2}(z)
$$

where the Dirac matrices were taken to be

$$
\boldsymbol{\alpha}_{3}=\left(\begin{array}{cc}
0 & i \\
-i & 0
\end{array}\right), \quad \boldsymbol{\beta}=\left(\begin{array}{ll}
0 & 1 \\
1 & 0
\end{array}\right)
$$

and the potential $V$ was introduced in the so-called scalar coupling, so that it is added to the mass term $m \rightarrow m+V$. For simplicity $m$ will henceforth denote the effective mass thus obtained, that is the old mass plus the constant term of $V$. The other, vector, coupling is equivalent, as shown in [7], so that essentially the equation in question is just

$$
\psi^{\prime \prime}=\left(V^{2}+V^{\prime}-E^{2}\right) \psi=: r \psi
$$

The question of exact solutions of such an equation with a rational coefficient $r(z) \in \mathbb{C}(z)$ is best expressed in terms of field extensions of $\mathbb{C}(z)$. An extension including all the elementary functions, direct integration and some special transcendental functions is the generalized Liouvillian extension. It consists of a finite chain of differential function fields,

$$
F_{0} \subset F_{1} \subset \ldots \subset F_{n}
$$

such that each new field $F_{k+1}$ is obtained from the previous $F_{k}$ by adjoining an element $x_{k}$ which satisfies one of the three following conditions:

1. $x_{k}$ is algebraic over $F_{k}$,

2. $x_{k}$ is primitive, i.e. $x_{k}^{\prime} \in F_{k}$,

3. $x_{k}$ is the solution of $x^{\prime}=a x$, for some $a$ in $F_{K}$.

These fields are differential fields precisely so that there is some notion of the derivative $x_{k}^{\prime}$, although here it is simply the differentiation with regard to $z$ and the starting field $F_{0}$ is just $\mathbb{C}(z)$. If the solution of a given equation belongs to $F_{n}$ we might call it a Liouvillian solution and it is explicitly expressible by finite number of elementary operations like integrals, exponentials or solving algebraic equations. In other words, the equation in question is solvable/integrable if it has Liouvillian solutions.

A more detailed exposition of the above can be found in [8], and here it should be added that this particular notion of exact solution is intimately connected with the underlying group theory. Namely, one defines the differential Galois group of a differential equation as the group of automorphisms of the field to which the solutions belong, such that their action on the base field $F_{0}$ is trivial. Intuitively it means that the group permutes the solutions, but does not change the equation's coefficients. It then follows that the equation is solvable when the group is solvable. In case of the second order equations like (4) the group is an algebraic subgroup of $\mathrm{SL}(2, \mathbb{C})$, and there exists an algorithm contrived by Kovacic [9] for deciding on its integrability and finding the solutions (for another form of the algorithm see [10]). Also, most of the common equations of mathematical physics have been completely analyzed in this regard (see e.g. the monograph [11] for a collection of results), so the question is often that of finding a connection with what is already known. This will be the main route taken here, allowing to uncover a new, wide class of integrable potentials.

\section{RATIONAL POTENTIALS}

A naive generalization of the results of [7] is difficult for the following reason. When $V$ is polynomial, it only has one pole at infinity (and so has $r(z)$ ), and it just the Laurent expansion with finitely many terms. The polynomial's coefficients are then all we need for the analysis. A rational $V$ can be defined by a quotient of two polynomials, and 
although the denominator still has finitely many coefficients, they are not used directly. It is the poles that matter so $r(z)$ needs to be expanded around each one. One is therefore left with an arbitrary number of poles of arbitrary orders and coefficients with which to construct a general rational potential that could be analyzed effectively with Kovacic's algorithm.

Unfortunately, even the seemingly simplest case of adding just one pole to an integrable polynomial potential leads to only implicit integrability relations, so a full classification analogous to the polynomial case is impossible. To see this let us consider the following

$$
V(z)=\frac{\alpha}{z}+m+\lambda z
$$

with $\alpha \neq 0$. In general this yields the (biconfluent) Heun differential equation whose solvability is determined by algebraic relations defined only recursively - the subject of this section. Explicit analysis is possible whenever one of the other parameters is zero, and the equation reduces to a special case of the confluent hypergeometric one, which has countable families of Liouvillian solutions [11].

More specifically, for $\lambda=0$ Eq. (4) becomes a special case of the Whittaker equation, analyzed in detail in the next section. When $\lambda \neq 0$ but $m=0$ it reduces to the Laguerre equation

$$
u g^{\prime \prime}(u)+\left(\alpha+\frac{1}{2}-u\right) g^{\prime}(u)=\frac{E^{2}}{4 \lambda} g(u)
$$

where

$$
z^{-\alpha} \psi(z)=e^{-u / 2} g(u), \quad u=-\lambda z^{2} .
$$

The solutions are the Laguerre functions $L_{n}^{(\alpha-1 / 2)}(u)$ with $n=\frac{-E^{2}}{4 \lambda}$, and these are again expressible by confluent hypergeometric functions. They are Liouvillian if at least one of $n$ or $n+\alpha-\frac{1}{2}$ is an integer (with one solution polynomial for positive integer $n$ ). Additionally, $E=0$ always leads to an explicit solution of

$$
\psi=\exp \int V \mathrm{~d} z
$$

regardless of the form of the potential.

The generic case with all parameters non-zero can be slightly simplified with taking $\lambda= \pm 1$ or, in other words, rescaling as follows

$$
z \rightarrow z / \sqrt{|\lambda|}, m \rightarrow m \sqrt{|\lambda|}, \quad E \rightarrow E \sqrt{|\lambda|} .
$$

The reason for using absolute value is to keep the physical quantities real. This will make identifying the physical integrable cases easier.

Turning now to Kovacic's algorithm, the first case will be the only one to possible hold, and the solution must be of the form

$$
\psi=P(z) \exp \int \omega(z) \mathrm{d} z
$$

with rational $\omega$ and polynomial $P$. Denoting $\psi^{\prime} / \psi=: \theta$ and substituting into (4) we have

$$
\theta^{\prime}+\theta^{2}=V^{2}+V^{\prime}-E^{2}
$$

so that the poles of $V$ and $\theta$ must agree except that there could be additional simple poles of $\theta$ with residue 1 not appearing in $V$. Since $\theta=\frac{P^{\prime}}{P}+\omega$, the first term is needed precisely to account for those poles, and $\omega$ has to have the same polynomial part as $V$ (up to a sign) so that the expansions at infinity agree. Finally, the pole $\alpha / z$ requires that $\omega$ includes either $\alpha / z$ or $(1-\alpha) / z$, so that there are four possibilities all in all:

$$
\omega_{1,2}=\frac{\alpha}{z} \pm(m+\lambda z), \quad \omega_{3,4}=\frac{1-\alpha}{z} \mp(m+\lambda z) .
$$

The next step is to substitute the above into the equation in question and look for polynomial solutions for $P$ with 
its degree $d$ determined by the highest order term in each respective equation. The four subcases are thus

$$
\begin{aligned}
\text { 1. } & P^{\prime \prime}+2\left(\frac{\alpha}{z}+m+\lambda z\right) P^{\prime} & =2 \lambda d P, & & =-\frac{E^{2}}{2 \lambda} \\
\text { 2. } & P^{\prime \prime}+2\left(\frac{\alpha}{z}-m-\lambda z\right) P^{\prime} & =\left(\frac{4 \alpha m}{z}-2 \lambda d\right) P, & d & =\frac{E^{2}}{2 \lambda}-2 \alpha-1, \\
\text { 3. } & P^{\prime \prime}+2\left(\frac{1-\alpha}{z}-m-\lambda z\right) P^{\prime} & =\left(\frac{2 m}{z}-2 \lambda d\right) P, & & =\frac{E^{2}}{2 \lambda}-2, \\
\text { 4. } & P^{\prime \prime}+2\left(\frac{1-\alpha}{z}+m+\lambda z\right) P^{\prime} & =\left(\frac{2 m(2 \alpha-1)}{z}+2 \lambda d\right) P, & d & =-\frac{E^{2}}{2 \lambda}+2 \alpha-1 .
\end{aligned}
$$

Taking

$$
P=\sum_{l=0}^{d} p_{l} z^{l}
$$

the above can be stated as recurrence relations

$$
\begin{aligned}
\text { 1. } & l(l+2 \alpha-1) p_{l}+2 m(l-1) p_{l-1} & =2 \lambda(d-l+2) p_{l-2}, \\
\text { 2. } & l(l+2 \alpha-1) p_{l}-2 m(l+2 \alpha-1) p_{l-1} & =2 \lambda(l-d-2) p_{l-2}, \\
\text { 3. } & l(l+1-2 \alpha) p_{l}-2 m l p_{l-1} & =2 \lambda(l-d-2) p_{l-2}, \\
\text { 4. } & l(l+1-2 \alpha) p_{l}+2 m(l-2 \alpha) p_{l-1} & =2 \lambda(d-l+2) p_{l-2},
\end{aligned}
$$

with common initial conditions of

$$
p_{d+1}=0, \quad p_{d}=1 .
$$

They reflect the fact that $P$ can always be taken to be a monic polynomial, and its degree is exactly $d$. Consequently, the relations hold for $l \leq d+1$ and one can successively generate $p_{l}$, as functions of the parameters, down to $p_{0}$. The last but one relation, i.e. for $l=2$ gives us $p_{0}$ but there is one more with $l=1$ and only two terms $p_{1}$ and $p_{0}$ since for polynomials $p_{-1} \equiv 0$. This is the required condition for the existence of polynomial $P$. It is convenient, however, to consider the last relation as the definition of $p_{-1}$, which will be a function of $m, \lambda, \alpha$ and $d$, and then the condition $p_{-1}=0$ (equivalent to the two-term relation between $p_{1}$ and $p_{0}$ ) will yield the admissible values of parameters for which a polynomial $P$ exists, and hence the main equation is solvable. Since the whole problem is considered over the complex domain, all polynomials have the maximal number of roots, counting with multiplicities. When considered as polynomials in $m, p_{-1}$ is of degree $d+1$ provided that the middle term of the recurrence does not vanish. This particular way that $m$ enters also means that all the polynomials are either even or odd functions of $m$.

For each degree $d$ the relations (16) implicitly define $p_{-1}$ whose roots then provide the value of parameters leading to an integrable case, while the energy is determined by its relation with $d$ in each case. Of course, one is mostly interested in the real values of the parameters as they correspond to the physical case. This is the reason for approaching $p_{l}$ as polynomials in $m$ and $\alpha$ ( $\lambda$ can be scaled away, as mentioned before). The particular form of the recurrence makes those sequences define orthogonal polynomials in most cases (although this property is of no use here), and their zeros are then all real, simple and with the separation property

$$
a_{i}^{(k+1)}<a_{i+1}^{(k)}<a_{i+1}^{(k+1)},
$$

where $a_{i}^{(k)}$ is the $i$-th zero of the $k$-th polynomial. To see this in the first subcase note that

$$
p_{d-1}=-d m, a_{1}^{(d-1)}=0,
$$

and

$$
p_{d-2}=d\left(2(d-1) m^{2}+1-2 \alpha-d\right) / 4, \quad a_{1}^{(d-2)}=-\sqrt{\frac{d+2 \alpha-1}{2(d-1)}}, a_{2}^{(d-2)}=\sqrt{\frac{d+2 \alpha-1}{2(d-1)}},
$$

so that distinct real roots exist for $2 \alpha>1-d . d=0$ is ruled out by $E \neq 0, d=1$ ruled out by $m \neq 0$, so the smallest $d$ is 2 , which translates to $\alpha>-1 / 2$. Assuming that the separation property holds for polynomials $p_{l}$ and $p_{l-1}$ the proof is by induction on $l$. Evaluating $p_{l-2}$ at a zero of $p_{l-1}$ gives

$$
l(l+2 \alpha-1) p_{l}\left(a_{i}^{(l-1)}\right)=-2(d-l+2) p_{l-2}\left(a_{i}^{(l-1)}\right),
$$


where $\lambda$ was taken negative, as is necessary by the energy condition in (14). Since the zeros of $p_{l}$ lie only between those of $p_{l-1}$ it follows that the left-hand side of the above is non-zero and switches sign as $i$ increases, provided that $l+2 \alpha-1$ is non-zero. It follows that $p_{l-2}$ must change sign in each interval $\left(a_{i}^{(l-1)}, a_{i+1}^{(l-1)}\right)$ which can happen only at a root. The only thing left to show that $p_{l-2}$ has two roots outside of $\left(a_{1}^{(l-1)}, a_{d-l+1}^{(l-1)}\right)$. With the further assumption that $l+2 \alpha-1>0$ the sign of $p_{l-2}$ at the last root of $p_{l-1}$ is opposite to the sign of $p_{l}$ but they both have the same sign in their leading powers of $m$ which are $m^{d+1}$ and $m^{d-1}$ respectively, so that $p_{l-2}$ must have another root for the signs to agree as $m \rightarrow \infty$. The same happens at the lowest root. Finally, care has to be taken as to the middle term of the recurrence, as it cannot vanish (the number of roots must be maximal). Fortunately for $l=1$ the relation just states that $\alpha p_{1}=2 \lambda(d+1) p_{-1}$ so effectively we can take $l \geq 3$ and $p_{1}=0$ as the integrability condition, and automatically the smallest $d$ to consider is 3 as $d=2$ implies $p_{-1}=-2 m$. Altogether this means that the induction is valid for $\alpha>-1$, and the separation property holds up to $p_{1}$.

The third subcase is analogous and even simpler, with the only difference being that the energy condition requires $\lambda>0$, and the positivity of $l+2 \alpha-1$ is enough to guarantee the appropriate changes of signs, and maximal degree of each $p_{l}$. For the first induction step, the condition for $p_{d-2}$ to have simple real roots is that $d-2 \alpha+1$ be positive. Since $d=0$ gives $p_{-1}=m$, it follows that $\alpha>1$ is enough for the separation property.

Subcases two and four differ in that the sign of $\lambda$ is now arbitrary, but the general proofs are the same and we get $\lambda>0, \alpha>0$ or $\lambda<0, \alpha<-d / 2$ in subcase two, and $\lambda>0, \alpha>(d+1) / 2$ or $\lambda<0, \alpha<1 / 2$. Where again the trivial cases $m=0$ were discarded. However, the results can be slightly strengthened here. In subcase two, for $\alpha=0$ the relation with $l=1$ just gives $p_{-1} \equiv 0$, and one can see that for $-1 / 2<\alpha<0$, the induction can still be carried as before, with the modification that signs at the last step are reverted. Namely

$$
2 \alpha p_{1}-4 m \alpha p_{0}=-2 \lambda(d+1) p_{-1},
$$

so that for $\lambda>0$ at the maximal root of $p_{0}$ the signs of $p_{-1}$ and $p_{1}$ are the same, but additionally the leading coefficients differ in sign, as the coefficient multiplying $m p_{0}$ in the relation is positive, while it was negative for all higher $l$. Thus the existence of the highest root follows. For $\lambda<0$ this cannot be done, as the main restriction comes from the roots of $p_{d-2}$ not from the third induction step.

The same is true for subcase four with $(d+1) / 2>\alpha>d / 2$ and $\lambda>0$ so that the region where the separation property holds can be extended to $\alpha>d / 2$. The case of $\alpha=(d+1) / 2$ is, by the energy condition, equivalent to $E=0$ so it brings no new solutions.

Putting the above together, and discarding the $m=0$ case we have

Theorem 1 The integrability condition $p_{-1}=0$, considered as an equation in $m$ has the full number of single real roots in the subcases (16) for

1. $\lambda<0$ and $\alpha>-1$. There are $\lfloor(d-1) / 2\rfloor$ positive roots with $d=-\frac{E^{2}}{2 \lambda}$.

2. $\lambda>0$ and $\alpha>-\frac{1}{2}$, or $\lambda<0$ and $\alpha<-d / 2$. There are $\lceil d / 2\rceil$ positive roots with $d=\frac{E^{2}}{2 \lambda}-2 \alpha-1$.

3. $\lambda>0$ and $\alpha<1$. There are $\lceil d / 2\rceil$ positive roots with $d=\frac{E^{2}}{2 \lambda}$.

4. $\lambda>0$ and $\alpha>d / 2$, or $\lambda<0$ and $\alpha<\frac{1}{2}$. There are $\lceil d / 2\rceil$ positive roots with $d=-\frac{E^{2}}{2 \lambda}+2 \alpha-1$.

Recall that \lfloor\rfloor and \lceil\rceil are the floor and ceiling functions, respectively.

Next, the inspection of the $m=0$ case tells us that for odd $d=2 n+1$ the conditions read

1. $p_{-1}=\prod_{n=0}^{\frac{1}{2}(d-1)} \frac{(2 n+1)(\alpha+n)}{\lambda(d-2 n+1)} \quad=0$,

2. $p_{-1}=\prod_{n=0}^{\frac{1}{2}(d-1)} \frac{(2 n+1)(\alpha+n)}{\lambda(2 n-d+3)} \quad=0$,

3. $p_{-1}=\prod_{n=0}^{\frac{1}{2}(d-1)} \frac{(2 n+1)(n+1-\alpha)}{\lambda(2 n-d+3)}=0$,

4. $p_{-1}=\prod_{n=0}^{\frac{1}{2}(d-1)} \frac{(2 n+1)(n+1-\alpha)}{\lambda(d-2 n+1)}=0$, 
so that there are always $n=(d-1) / 2$ real simple roots. This happens because the recurrence involves only two terms then, and we start with $p_{d}$ reducing the index by 2 at each iteration to get to $p_{-1}$. It then follows that in some neighborhood of $m=0$ there must still be $n$ real simple roots, because $p_{-1}$ is a polynomial in $\alpha$ whose coefficients are again polynomials of $m$, so that the roots must be continuous functions of $m$. Note however, that in subcases 2 and 4, the full polynomial could be of higher degree in $\alpha$, thanks to the middle term, and there will be additional roots, that go to infinity when $m \rightarrow 0$.

The analysis of even degrees is much more involved, so we include the proof in the appendix, and just state the general result here

Theorem 2 In the vicinity of $m=0$ there is always the maximal number of real simple roots in $\alpha$ of the integrability condition $p_{-1}=0$. Depending on the parity of $d$ and the subcases of (16) one has

1. For odd $d$ there are $(d+1) / 2$ roots (including $\alpha=0$ ) given by (23). For even $d$ there are $d / 2$ roots interleaved with the roots of (A3).

2. For odd $d$ there are $(d+1) / 2$ roots (including $\alpha=0$ ) given by (23). For even $d$ there are $d / 2$ roots interleaved with the roots of (A19) (also including $\alpha=0$ ).

3. For odd $d$ there are $(d+1) / 2$ roots given by (23). For even $d$ there are $d / 2$ roots interleaved with the roots of (A15).

4. For odd $d$ there are $(d+1) / 2$ roots, one of them being $\alpha=(d+1) / 2$, given by (23). For even $d$ there are $d / 2$ roots interleaved with the roots of (A23), except for the the shared root $\alpha=(d+1) / 2$.

The the best way to view all the branches of the algebraic sets defined by the integrability conditions, and to get the intuition about the asymptotic behavior of roots, is a plot of $p_{-1}=0$ in the $(m, \alpha)$ plane shown in Figs. 1 and 2 ,
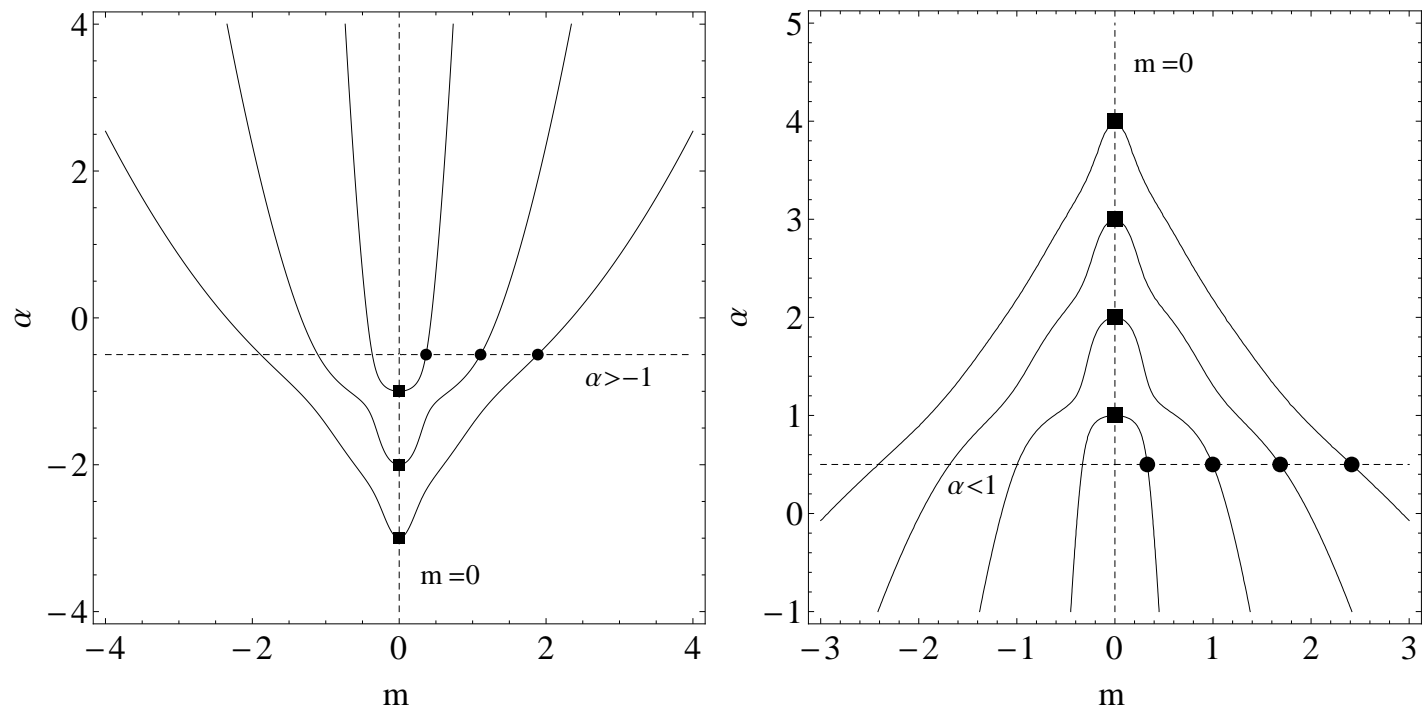

Figure 1: The curves (solid) defined by $p_{1}=0$ in subcases 1 and $p_{-1}=0$ in subcase 3, both with $d=7$. Circles represent zeroes obtained with the separation property, while squares are zeroes for $m=0$ that change into simple roots for small positive (and negative) $m$.

The graphs suggest that there are horizontal asymptotes in subcases 2 and 4 , so that for large $m$ there would also need to exist a fixed number of solutions. To check this, let us take rescaled polynomials defined by $p_{l}=m^{d-l} r_{l}$ which they satisfy

$$
\begin{aligned}
\text { 2. } \quad l(l+2 \alpha-1) \frac{r_{l}}{m^{2}}-2(l+2 \alpha-1) r_{l-1} & =2 \lambda(l-d-2) r_{l-2}, \\
\text { 4. } \quad l(l+1-2 \alpha) \frac{r_{l}}{m^{2}}+2(l-2 \alpha) r_{l-1} & =2 \lambda(d-l+2) r_{l-2},
\end{aligned}
$$



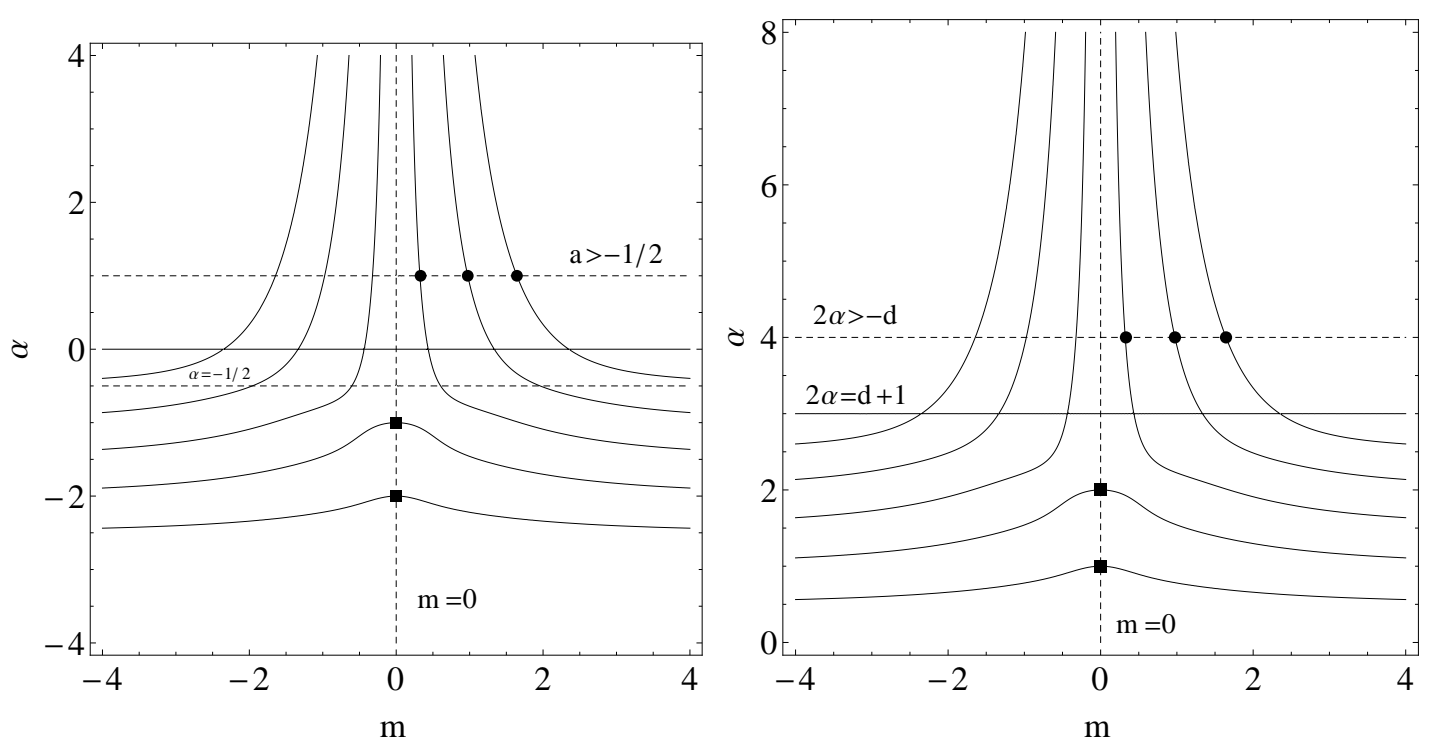

Figure 2: The curves (solid) defined by $p_{-1}=0$ in subcases 2 and 4 , both with $d=5$. Circles represent zeroes obtained with the separation property, while squares are zeroes for $m=0$ that change into simple roots for small positive (and negative) $m$.

where the rescaling is based on the maximal power of $m$ appearing in $p_{l}$. This means that each $r_{l}$ is finite as $m \rightarrow \infty$ and the limit case of the relations reads

$$
\text { 2. } \begin{aligned}
-2(l+2 \alpha-1) r_{l-1} & =2 \lambda(l-d-2) r_{l-2}, \\
\text { 4. } \quad 2(l-2 \alpha) r_{l-1} & =2 \lambda(d-l+2) r_{l-2},
\end{aligned}
$$

so that at each recurrence step a real zero in $\alpha$ is introduced - exactly as in the $m=0$ case above. In subcase 2 $l=1$ adds the precluded $\alpha=0$, while in subcase $4, l=d+1$ is the previously mentioned straight line of solutions (independent of $m$ ). These real roots at infinity cannot turn into complex one for large finite $m$ since any complex roots would have to come in conjugate pairs. This would require the polynomials of twice the degree, but also the roots at infinity to be double. By continuity we thus obtain

Theorem 3 For sufficiently large $m^{2}$ there are always solutions of $p_{-1}=0$, when considered as an equation in $\alpha$. In subcase 2, there are $d$ solutions which asymptotically approach $\alpha=(1-l) / 2$ for $l=2, \ldots, d+1$. In subcase 4 , there are $d$ solutions that approach $\alpha=l / 2$ for $l=1, \ldots, d$ and the line $\alpha=(d+1) / 2$ is also always a solution.

The question remains as to the vertical asymptotics of the branches, that is what happens for infinite $\alpha$. Rescaling this time by $p_{l}=\alpha^{d-l} r_{l}$ gives

$$
\begin{aligned}
& \text { 2. }-4 m r_{l-1}=2 \lambda(l-d-2) r_{l-2}, \\
& \text { 4. }-4 m r_{l-1}=2 \lambda(d-l+2) r_{l-2},
\end{aligned}
$$

so that only the factor of $m$ is introduced at each step. This means that all the branches tend to $m=0$. For any straight line of constant $m$, each branch must intersect it then, as the previous theorem guarantees that they extend towards infinite $m$. If in the vicinity of $m=0$ it were possible to prove the existence of solutions also for odd degrees, that would translate into global existence of exactly $d+1$ roots in $\alpha$ for any value of $m$ (counting also $\alpha=0$ and $\alpha=(d+1) / 2$ where necessary $)$.

Finally, the last two cases of Kovacic's algorithm need to be dealt with. The second case cannot hold for the required quantities $E_{c}$ as defined in [9] are: $E_{0}=\{4(1-\alpha), 2,4 \alpha\}$ and $E_{\infty}=\{-2\}$. Then the degree of the polynomial $P$ defined as above should be $d=\left(e_{\infty}-e_{0}\right) / 2$ for $e_{x} \in E_{x}$, and this implies that $\alpha$ has to be an integer or half integer. However this makes all $e_{x}$ even, so that case two cannot hold. Case three is ruled out because the order of $r(z)$ at infinity is less than 2 . In other words infinity is an irregular singular point.

\section{RECONSTRUCTION OF THE POTENTIAL}

The direct approach above gave us only implicit conditions and with so many general classes of integrable equations it is natural to try and work ones way in the other direction, i.e. reconstructing the integrable potentials. To 
demonstrate the idea let us first consider how to generalize the basic form of the equation in question.

We want our main equation (4) to assume some particular, prescribed form with rational $r(z)$, and notice immediately that energy enters in such a way that the infinity must be an irregular singular point. This could in principle be remedied by other parameters of $V$, but only for one fixed value of $E^{2}$, while the physical nature of the problem allows any $E$ to begin with (and usually and with a restricted, but still infinite spectrum). It is thus better to assume that infinity is irregular, and the special values of parameters for which it is not will up in the analysis anyway.

At first one might ask whether there are rational potentials with the property that only the infinity is a singular point - like in the polynomial case. $V(z)$ would then need at least one pole, not to be polynomial, and it could only be of first order and residue 1 if $V^{2}+V^{\prime}$ is to have no poles. The general potential would then be

$$
V(z)=\sum_{k=1}^{N} \frac{1}{z-c_{k}}+R(z)
$$

with a polynomial $R(z)$, and $r(z)$ reads

$$
r(z)=R^{2}+R^{\prime}+2 R \sum_{k=1}^{N} \frac{1}{z-c_{k}}+2 \sum_{\substack{k, l=1 \\ k \neq l}}^{N} \frac{1}{\left(x-c_{k}\right)\left(x-c_{l}\right)}-E^{2} .
$$

The only possibility of removing the poles is cancellation between the two sums, and expanding them at each $c_{k}$ the condition is

$$
R\left(c_{k}\right)+\sum_{\substack{l=1 \\ l \neq k}}^{N} \frac{1}{c_{k}-c_{l}}=0
$$

The poles are all different (otherwise the residue would not be 1), so that this system of $N$ equations determines a polynomial of degree $N-1$ uniquely, but any other polynomial which vanishes at the poles can be added, so the most general form of $R$ is

$$
R(z)=R_{1}(z)+R_{2}(z) \prod_{k=1}^{N}\left(z-c_{k}\right)
$$

where $R_{1}$ satisfies (29) and $R_{2}$ is arbitrary. The system determining coefficients of $R_{1}$ has no explicit general solution (inverse of the Vandermond matrix) for arbitrary $N$, so let us again look at the potential with just one pole at zero so that

$$
R(z)=z R_{2}(z)=z \sum_{k=0}^{n} r_{k} z^{k}
$$

and

$$
r(z)=3 R_{2}+z R_{2}^{\prime}+z^{2} R_{2}^{2}-E^{2}
$$

With no poles in $r(z)$ and irregular infinity, only the first case of Kovacic algorithm can hold, so that the solution must be of the form (11). Substituting this into the differential equation (4) and comparing with $r(z)$ at infinity tells us that $\omega=z R_{2}$ and also that the degree of $P$ must be 1 . Taking the polynomial to be monic $P(z)=z+p_{0}$ results in the final equation

$$
\left(E^{2}-2 r_{2}\right) p_{0}+E^{2} z=0
$$

which can hold only when $R_{2}$ is of degree 1 with $p_{0}=r_{0} / r_{1}$ and $E^{2}=2 r_{0}\left(r_{1}=0\right.$ leads to $E=0$ again $)$. The integrable potential is then

$$
V(z)=\frac{1}{z}+z\left(r_{0}+r_{1} z\right)
$$

and the solutions

$$
\begin{aligned}
& \psi_{1}(z)=\left(r_{1} z+r_{0}\right) \exp \left(z^{2}\left(3 r_{0}+2 r_{1} z\right) / 6\right) \\
& \psi_{2}(z)=\psi_{1} \int \frac{1}{\psi_{1}(z)^{2}} \mathrm{~d} z
\end{aligned}
$$


For higher $N$ it is possible to proceed halfway in that the expansion of $r(z)$ at infinity implies that $\omega=R(z)$, and the solution of the prescribed form $P \exp \int \omega$ leads to

$$
P^{\prime \prime}+2 R P^{\prime}+\left(R^{2}+R^{\prime}-r\right) P=0 .
$$

The highest order term vanishes when $\operatorname{deg} P=N$, so this is just a linear system for the coefficients of $P(z)$, but to write it out explicitly one would have to know $R_{1}$, which amounts to solving (29) separately for each value of $N$.

\section{WHITTAKER EQUATION}

Finally, let us look at a known type of equation for which non-trivial integrable cases exist. In contrast to the previous example, we expect $r(z)$ to have a pole but want to restrict the degree at infinity (the polynomial part). As mentioned before, there is a special case of Heun equation which reduces to the confluent hypergeometric one and has a finite pole of order two in $r(z)$, while only the energy accounts for infinity being irregular. Its general form is the Whittaker equation:

$$
y^{\prime \prime}=\left(\frac{1}{4}-\frac{\kappa}{z}+\frac{4 \mu^{2}-1}{4 z^{2}}\right) y,
$$

and since its solvability conditions are known, we can find out which potentials allow Liouvillian solutions.

In order for the Dirac equation to assume such form, the potential has to satisfy

$$
V^{2}+V^{\prime}-E^{2}=\left(\frac{1}{4}-\frac{\kappa}{z}+\frac{4 \mu^{2}-1}{4 z^{2}}\right)
$$

which is a Riccati equation. Its rational solution can be obtained by the analysis of the poles of the right-hand side. The general solution to the above is

$$
V=m+\frac{\alpha}{z}+\sum_{k=1}^{d} \frac{1}{z-c_{k}} .
$$

The poles $c_{k}$ have to be included even though they do not correspond to the poles of $V^{2}+V^{\prime}$ because each term of the sum is a solution to $V^{2}+V^{\prime}=0$. Since the pole at zero is essential in the equation, its residue is $\alpha \neq 1$. Alternatively, the above could be rewritten as

$$
V=m+\frac{\alpha}{z}+\frac{P^{\prime}(z)}{P(z)}
$$

where $P$ is a polynomial with $d$ distinct roots $c_{k}$, such that $P(0) \neq 0$, and can be taken to be monic.

Substituting this form of $V$ to the coefficient $r$, one gets

$$
r=m^{2}-E^{2}+\frac{\alpha(\alpha-1)}{z^{2}}+\frac{2 m \alpha}{z}+\frac{2(m z+\alpha) P^{\prime}+z P^{\prime \prime}}{z P} .
$$

The restrictions on the singular points require that the last term is of the form $a+b / z+c / z^{2}$, so that we have an equation for $P$

$$
z P^{\prime \prime}+2(\alpha+m z) P^{\prime}=\left(a z+b+\frac{c}{z}\right) P .
$$

As the solution is supposed to be a polynomial, the coefficient $a$ must be zero, or the terms of the highest order would not cancel. Similarly, the lowest order terms require that $P(0) c=0$ and since we excluded zero from the list of the roots $c$ also must vanish. We now have

$$
z P^{\prime \prime}+2(\alpha+m z) P^{\prime}=b P
$$

which is the associated Laguerre equation, with polynomial solutions only for

$$
b=2 m d, \quad d \in \mathbb{N}, m \neq 0,
$$


so that

$$
P=L_{d}^{2 \alpha-1}(-2 m z)
$$

With such condition, the Dirac equation becomes

$$
\psi^{\prime \prime}=\left(m^{2}-E^{2}+\frac{\alpha(\alpha-1)}{z^{2}}+\frac{2 m(\alpha+d)}{z}\right) \psi
$$

and assuming $m^{2} \neq E^{2}$ (the special case will be dealt with below) a change of the dependent variable to

$$
u=2 \sqrt{m^{2}-E^{2}} z
$$

gives

$$
\psi^{\prime \prime}(u)=\left(\frac{1}{4}+\frac{\alpha(\alpha-1)}{u^{2}}+\frac{m}{\sqrt{m^{2}-E^{2}}} \frac{\alpha+d}{u}\right) \psi,
$$

so that the appropriate Whittaker's equation's coefficients are

$$
\begin{aligned}
& \mu=-\left(\alpha-\frac{1}{2}\right), \\
& \kappa=-\frac{m}{\sqrt{m^{2}-E^{2}}}(\alpha+d),
\end{aligned}
$$

and we simply have

$$
\psi(z)=W_{\kappa, \mu}\left(2 \sqrt{m^{2}-E^{2}} z\right) .
$$

Since the equation is of the second order, there will be also another, linearly independent solution given by $W_{-\kappa, \mu}(-z)$. However, in application one will choose only one taking into account the physical context such as square integrability, and the domain of $z$. For example, in the $z \gg 0$ region, one would not take the function $W_{-\kappa, \mu}(-z)$ since it behaves as $\exp (z)$ asymptotically, while $W_{\kappa, \mu}(z)$ behaves as $\exp (-z)$.

Here, however, we are interested in the general nature of the involved functions, rather than particular physical model, so we wish to impose other restrictions, namely the Liouvillian character of the solutions. For the Whittaker equation the conditions for that are well known [11] and here they give

$$
\begin{aligned}
& p:=\mu+\kappa-\frac{1}{2} \in \mathbb{Z}, \text { or } \\
& q:=\mu-\kappa-\frac{1}{2} \in \mathbb{Z},
\end{aligned}
$$

which, upon substitution, leads to

$$
\begin{aligned}
p+1 & =\frac{m}{\sqrt{m^{2}-E^{2}}}(\alpha+d)+\alpha \in \mathbb{Z}, \text { or } \\
q & =\frac{m}{\sqrt{m^{2}-E^{2}}}(\alpha+d)-\alpha \in \mathbb{Z} .
\end{aligned}
$$

In other words, when the potential is such that $\alpha=-d$ the solutions are always Liouvillian, and otherwise the parameters must satisfy

$$
\frac{m}{\sqrt{m^{2}-E^{2}}}=\frac{l \pm \alpha}{d+\alpha}, \quad l \in \mathbb{Z},
$$

which in turn means that the integrable part of the spectrum is bounded by

$$
0 \leq \frac{E^{2}}{m^{2}}=1-\left(\frac{d+\alpha}{l \pm \alpha}\right)^{2} \leq 1 .
$$

Note that when $\alpha$ is an integer itself, the case of $l= \pm \alpha$ is excluded by $m \neq 0$. 
For the particular case of $m^{2}=E^{2}$, Eq. (46) is effectively the Bessel equation

$$
w^{2} \varphi^{\prime \prime}(w)+w \varphi^{\prime}(w)+\left(w^{2}-(1-2 \alpha)^{2}\right) \varphi(w)=0,
$$

when the variables are changed according to

$$
\psi(z)=\sqrt{z} \varphi(w), \quad w^{2}:=-8 m(\alpha+d) z,
$$

so, assuming $\alpha \neq d$, the solutions are given in term of the Bessel functions

$$
\psi(z)=\sqrt{z} J_{1-2 \alpha}(2 \sqrt{-2 m(\alpha+d) z}),
$$

and these are known to be Liouvillian only if their order, i.e. $1-2 \alpha$, is half of an odd integer.

Finally, for $\alpha=-d$ (and $m^{2}=E^{2}$ ), Eq. (46) is solvable by elementary functions of

$$
\psi(z)=c_{1} z^{\alpha}+c_{2} z^{1-\alpha} .
$$

\section{SUMMARY}

To put the results together, we have shown that the one-dimensional Dirac equation with a rational potential will lead to integrability conditions which in general will be given by polynomials defined recursively. Also, that rational potentials can be determined if some natural conditions are required of the resulting equation itself. The direct and inverse results are as follows.

The simplest form of $V=\alpha / z+m+\lambda z$ gives rise to the biconfluent Heun equation (if none of the parameters is zero). Its solvability is determined by the polynomial condition $p_{-1}(\alpha, m)=0$, obtained by one of the relations (16) (together with the respective conditions on $E, \lambda$ and $d$ given by (14)) - if any of the four can be solved it gives a Liouvillian solution. However, we are only interested in real values of the parameters and the three theorems 1, 2 and 3 characterize (to some extent) the position and asymptotics of the real roots.

The special cases of the above potential, for vanishing parameters are

1. $\alpha=0$, the Dirac oscillator solvable by Hermite functions [7].

2. $m=0$, the Laguerre equation, solvable with $L_{n}^{\alpha-1 / 2}(\sqrt{-\lambda} z)$, with $4 \lambda n=-E^{2}$.

(These are Liouvillian functions when $n$ or $n+\alpha-1 / 2$ is an integer.)

3. $\lambda=0$, the Whittaker equation of section $\mathbf{V}$, summarized below.

If we restrict the problem somewhat by requiring that $r(z)$ remains a polynomial, although $V(z)$ might still be rational, we obtain a general condition that the potential is of the form

$$
V(z)=\sum_{k=1}^{N} \frac{1}{z-c_{k}}+R_{1}(z)+R_{2}(z) \prod_{k=1}^{N}\left(z-c_{k}\right),
$$

with $R_{1}$ of degree $N-1$ such that

$$
R_{1}\left(c_{k}\right)+\sum_{\substack{l=1 \\ l \neq k}}^{N} \frac{1}{c_{k}-c_{l}}=0,
$$

and $R_{2}$ and arbitrary polynomial. This is the most general rational potential that leads to a polynomial coefficient $r(z)$. Due to $R_{1}$ being given only implicitly (although uniquely) only the one-pole case in analyzed in section IV. The potential turns out to be

$$
V(z)=\frac{1}{z}+r_{0} z+r_{1} z^{2}
$$

and the integrable values of the energy are $E^{2}=2 r_{0}$.

Finally, choosing a predetermined $r(z)$ which is simpler than in the Heun equation, but still has infinity as an irregular singular point and one other pole, leads to the Whittaker (confluent hypergeometric) equation. The potential becomes

$$
V=m+\frac{\alpha}{z}+\frac{P^{\prime}(z)}{P(z)}
$$


where $P$ is expressed by the associated Laguerre polynomial of degree $d$ as

$$
P(z)=L_{d}^{2 \alpha-1}(-2 m z)
$$

and $m$ is necessarily non-zero. The solutions are then given by the Whittaker functions $W_{\kappa, \mu}(z)$, and are additionally Liouvillian (can be expressed in closed form) when

1. $\alpha=-d$ regardless of energy, or

2. $2 \alpha \in \frac{1}{2}+\mathbb{Z}$ for $E^{2}=m^{2}$,

3. for particular levels satisfying $\frac{m}{\sqrt{m^{2}-E^{2}}}(\alpha+d) \pm \alpha \in \mathbb{Z}$.

The third possibility gives countably infinitely many energetic levels which are confined to $E \in[-m, m]$. All the cases are put together in table 1

\begin{tabular}{|c|lll|}
\hline$\alpha$ & $E \in \mathbb{C}$ & $\frac{m}{\sqrt{m^{2}-E^{2}}}(\alpha+d) \pm \alpha \in \mathbb{Z}$ & $E^{2}=m^{2}$ \\
\hline$\alpha \in \mathbb{C}$ & $W_{\kappa, \mu}(u), W_{-\kappa, \mu}(-u)$ & $W_{\kappa, \mu}(u), W_{-\kappa, \mu}(-u)[L]$ & $\sqrt{z} J_{1-2 \alpha}(w), \sqrt{z} Y_{1-2 \alpha}(w)$ \\
$2 \alpha-\frac{1}{2} \in \mathbb{Z}$ & $W_{\kappa, \mu}(u), W_{-\kappa, \mu}(-u)$ & $W_{\kappa, \mu}(u), W_{-\kappa, \mu}(-u)[L]$ & $\sqrt{z} J_{1-2 \alpha}(w), \sqrt{z} Y_{1-2 \alpha}(w)[L]$ \\
$\alpha=-d$ & $\sqrt{z} J_{\alpha-\frac{1}{2}}\left(\frac{u}{2 i}\right), \sqrt{z} Y_{\alpha-\frac{1}{2}}\left(\frac{u}{2 i}\right)[L]$ & $\sqrt{z} J_{\alpha-\frac{1}{2}}\left(\frac{u}{2 i}\right), \sqrt{z} Y_{\alpha-\frac{1}{2}}\left(\frac{u}{2 i}\right)[L]$ & $z^{\alpha}, z^{1-\alpha}[L]$ \\
\hline
\end{tabular}

Table I: The list of bases of solutions depending on parameters. $W$ denotes the Whittaker function, $J$ and $Y$ the Bessel functions. The auxiliary variables are: $w=2 \sqrt{-2 m(\alpha+d) z}, u=2 \sqrt{m^{2}-E^{2}} z$. [L] signifies a Liouvillian pair of solutions.

It should be clear from the general expression of the potential (62) that it describes a very wide class, with an arbitrary number of singularities, thus modeling drastically different physical situations. The mathematical analysis carried out here gives the full information about the integrability and formal solutions, but it is beyond its scope to select the appropriate eigenstates and respective energies corresponding to a particular quantum system. However, just as is the case with the Dirac oscillator, the integrability condition can be thought of as some quantization rule. As shown in [7] dual rules will hold for the vector coupling upon exchanging the roles of $E$ and $m$. Either way, one can speak of some form of quasi-integrability here, since Liouvillian solutions depend on values of (at least) two independent physical parameters, so even before taking the physical restrictions into account, we are left with at most countably many explicit solutions.

Concerning the property of shape invariance, the recovered potential contains the known Coulomb case (albeit the spatial variable is then radial) listed in [3]. This is the only case when the property holds, so that the whole class is in fact new, and the method described can be used to uncover more solvable cases by loosening some of the initial requirements. Future generalizations might include target equations with more singular (regular or not) points, or simply integrability conditions for given, more complex potentials (trigonometric, meromorphic etc.).

Of course the Liouvillian solutions are not the only notion of integrability, and most linear equations can be solved by means of series or numerically, but the concept of an exact solution remains as the starting point in any such approach. Any further study of the properties of the system in question, especially qualitative, it greatly facilitated by working on explicit formulae, which this work hopefully provides.

\section{Acknowledgments}

This research has been supported by grant No. DEC-2011/02/A/ST1/00208 of National Science Centre of Poland.

\section{Appendix A}

When the degree $d=2 n$ is even, $p_{-1}$ will be an odd polynomial, so $m=0$ will make it trivially zero. Also, writing the relation for even $l$ and taking the limit we get (in the first subcase)

$$
l(l+2 \alpha-1) p_{l}=-2(d-l+2) p_{l-2},
$$

so that only even indexed polynomials are generated from $p_{d}=1$, leading to $p_{0}$ but not $p_{-1}$. It is thus necessary to consider polynomials $r:=p_{l} / m$, which changes the relation to

$$
l(l+2 \alpha-1) r_{l}+2(l-1) p_{l-1}=-2(d-l+2) r_{l-2},
$$


Since $m$ does not enter the relation, the limit of $m \rightarrow 0$ changes only the initial polynomial $r_{d-1}=-d$. Note that $r_{l}$ is defined only for odd $l$ and $p_{l-1}$ is the same as above, with its index even. The non-vanishing of energy forces $d$ to be at least 2 , by (14), and then $r_{-1}=2 \alpha / 3$, so there is one simple root (albeit excluded).

The $d \geq 4$ case can be dealt with inductively. First the explicit formula for the even indexed polynomials is

$$
p_{l}=\prod_{k=1+l / 2}^{d / 2} \frac{-2 k(\alpha+k-1 / 2)}{d-2 k+2}
$$

Since $p_{d-2}=-d(2 \alpha+d-1) / 4$ has only one root, and as $l$ decreases, there appear new simple roots at half (negative) integers, given by

$$
\alpha_{k}=\frac{1}{2}-k, \quad k=\frac{d}{2}, \ldots, 1+\frac{l}{2}
$$

it follows that $p_{l}$ all share the first zero, and each has $(d-l) / 2$ zeroes altogether. The sign of the polynomial does not change past its final zero, and can be determined from (A3)

$$
\operatorname{sgn}\left(p_{l}\left(\alpha_{l, M}+1 / 2\right)\right)=(-1)^{(d-l) / 2},
$$

where the subscript in $\alpha_{l, M}$ indicates that the maximal root depends on $l$, and the reason for taking the value specifically at $\alpha_{l, M}+1 / 2$ will become clear in a moment.

The initial inductive step will be $l=d-1$ with the relation

$$
(d-1)(d+2 \alpha-2)(-d)+2(d-2) p_{d-2}=-6 r_{d-3},
$$

where $r_{d-1}=-d$, and the other polynomials are linear in $\alpha$ but it is more instructive not to substitute them explicitly. Now, at the first (and only) root of $p_{d-2}, \alpha_{d / 2}$, the above simply reduces to

$$
d(d-1)=-6 r_{d-3}\left(\alpha_{d / 2}\right)>0,
$$

whereas at $\alpha_{d / 2}+\frac{1}{2}$ we have $\left(d+2 \alpha_{d / 2}-1\right)=0$ so

$$
2(d-2) p_{d-2}\left(\alpha_{d / 2}+1 / 2\right)=-6 r_{d-2}\left(\alpha_{d / 2}+1 / 2\right) .
$$

By (A5), the above translates to

$$
(-1)^{(d-d+2) / 2}=-1=-\operatorname{sgn}\left(r_{d-2}\left(\alpha_{d / 2}+1 / 2\right)\right)=-\operatorname{sgn}\left(r_{d-2}\left(\alpha_{d / 2}+1\right)\right)<0,
$$

where the last equality follows from the fact, that $r_{d-2}$ is linear (by construction) and already changes sign once in the interval $\left(\alpha_{d / 2}, \alpha_{d / 2}+\frac{1}{2}\right)$. Consequently there is a root, and since $\alpha_{d / 2}+1$ is the next zero in the sequence (A4), we know the signs of $r_{d-3}$ at the first two $\alpha_{k}$.

The next step is to show that all following $r_{l}$ have the same signs at the $(d-l+1) / 2$ roots of $p_{l-1}$, in alternating sequence, and that there is an additional change of sign at the $(d-l) / 2$-th root of $p_{l-3}$. This is to extend the previous paragraph, where the signs agreed at the first root, and there was a change at the next one. For $l<d-1$ the relation at any zero $\alpha_{k}$ of $p_{l-1}$ is

$$
l(l-2 k) r_{l}\left(\alpha_{k}\right)=-2(d-l+2) r_{l-2}\left(\alpha_{k}\right),
$$

so that the signs agree because $2+l<2 k<d$. Thus as the signs alternate for $r_{l}$ they also alternate for $r_{l-2}$ indicating $(d-l+1) / 2$ simple zeroes. Note that at the first zero $\alpha_{d / 2}$, the signs of all $r_{l}$ are thus -1 and accordingly $\operatorname{sgn}\left(r_{l-2}\left(\alpha_{l-1, M}\right)\right)=(-1)^{(d-l+1) / 2}$. As before we look what happens past the maximal zero at $\alpha_{l-1, M}+1 / 2=(1-l) / 2$ so that the first term of the relation is zero and

$$
2(l-1) p_{l-1}\left(\alpha_{l-1, M}\right)=-2(d-l+2) r_{l-2}\left(\alpha_{l-1, M}\right),
$$

or, in term of signs,

$$
(-1)^{(d-l+1) / 2}=-\operatorname{sgn}\left(r_{l-2}\left(\alpha_{l-1, M}+1 / 2\right)\right) .
$$

Because $r_{l-2}$ is a sum of polynomials of degree $(d-l+1) / 2$, its degree cannot be higher, and the last change of sign means this is strictly the degree with all roots real and simple. With no more sign changes it also follows that $r_{l-2}\left(\alpha_{l-1, M}+1\right)=(-1)^{(d-l+1) / 2}$ and since that is the last root of $p_{l-3}$ the last induction step is completed. 
The last polynomial, $r_{-1}$, must thus have $d / 2$ real simple zeroes, although the last one is $\alpha=0$, for, as mentioned before, $p_{-1}$ is proportional to $\alpha p_{1}$.

The third subcase is very similar to the first one, with positive $\lambda$ this time, and the even $p_{l}$ characterized by

$$
\begin{gathered}
p_{l}=\prod_{k=1+l / 2}^{d / 2} \frac{k(2 \alpha-2 k-1)}{d-2 k+2}, \\
\alpha_{k}=k+1 / 2, \\
\operatorname{sgn}\left(p_{l}\left(\alpha_{l, m}-1 / 2\right)\right)=(-1)^{(d-l) / 2},
\end{gathered}
$$

where $\alpha_{l, m}$ is the smallest root, whereas the greatest root is shared by all $p_{l}$. For odd $l$ the relation reads

$$
l(l+1-2 \alpha) r_{l}-2 l p_{l-1}=2(l-d-2) r_{l-2},
$$

and exactly as above the signs of $r_{l}$ and $r_{l-2}$ agree on zeroes of $p_{l-1}$. The smallest root of $p_{l-1}$ is $1+l / 2$ so the relation at $\alpha_{l-1, m}-1 / 2$ gives

$$
l p_{l-1}\left(\alpha_{l-1, m}-1 / 2\right)=(d-l+2) r_{l-2}\left(\alpha_{l-1, m}-1 / 2\right) .
$$

We can then proceed inductively, as before, by noting that $r_{d-1}=d$ so that all $r_{l}$ are positive at the maximal root, and they alternate signs at $\alpha_{k}$, with the additional change of sign resulting from the above equation. The only trivial case to discard is $d=0$ for which $r_{-1}=1$, while for $d \geq 2$ it follows that there exist $d / 2$ real simple roots.

Subcase 2 differs in two ways. Firstly, the sign of $\lambda$ is arbitrary, but this only means that for $\lambda<0$ the signs of subsequent $r_{l}$ will be opposite at zeroes of $p_{l}$ instead of the same. That will not change the fact that the signs alternate and account for all but one zero. Here is where the second difference appears, for we have

$$
\begin{gathered}
p_{l}=\prod_{k=1+l / 2}^{d / 2} \frac{k(2 \alpha+2 k-1)}{2 k-d-2}, \\
\alpha_{k}=1 / 2-k
\end{gathered}
$$

and as the relation is now

$$
l(l+2 \alpha-1) r_{l}-2(l+2 \alpha-1) p_{l-1}=2 \lambda(l-d-2) r_{l-2}
$$

it means that at $\alpha_{l-1, M}+1 / 2$ we get

$$
0=2 \lambda(l-d-2) r_{l-2}((1-l) / 2)
$$

So that the last zero of $r_{l}$ is known explicitly and it ensures the desired changes of signs at roots of the appropriate $p_{l}$. Since $r_{d-1}=2 \alpha+d$ and $p_{d-2}=-d(2 \alpha+d-1) / 2$ have different roots (and hence the sign changes accordingly), induction on $l$ yields the existence of $1+d / 2$ roots of $r_{-1}$ with the explicit root being $\alpha=0$. Note that this also holds for $d=0$ where $r_{-1}=2 \alpha$.

Finally, in subcase 4 , there is a complication due to $r_{d-1}=-2 \alpha+d+1, p_{d-2}=d(-2 \alpha+d+1) / 4$, so that all polynomials share a root. As mentioned before, it corresponds to the degenerate $E=0$ case. This is the greatest of the roots of $p_{l}$ which are

$$
\begin{gathered}
p_{l}=\prod_{k=1+l / 2}^{d / 2} \frac{k(-2 \alpha+2 k+1)}{\lambda(d-2 k+2)}, \\
\alpha_{k}=k+1 / 2
\end{gathered}
$$

Let us consider $\lambda>0$ for clarity (the negative case is completely analogous). Note that $p_{l}$ will be positive for $\alpha$ less than the smallest zero because the leading coefficient will be $(-\alpha)^{(d-l) / 2}$ and since the relation is

$$
l(l+1-2 \alpha) r_{l}+2(l-2 \alpha) p_{l-1}=2(d-l+2) r_{l-2}
$$

the same will be true for $r_{l}$ and its smallest zero. We will see that apart from $\alpha=(d+1) / 2$ all the zeroes of $r_{l-2}$ lie between the zeroes of $p_{l-3}$. Direct computation shows that the smaller zero of $r_{d-3}$ is $3 d(d-1) /(6 d-4)$ which lies 
between $(d-1) / 2$ and $(d+1) / 2$. In particular $r_{d-3}$ is positive at $(d-1) / 2=\alpha_{d-4, m}$. Assume that this is true for $r_{l}$ and the relation for zeroes of $p_{l-1}$ other than $(d+1) / 2$ gives

$$
l\left(l+1-2 \alpha_{k}\right) r_{l}\left(\alpha_{k}\right)=2(d-l+2) r_{l-2}\left(\alpha_{k}\right),
$$

with the coefficient of $r_{l}$ being negative due to (A23). At $\alpha_{l-1, m}-1 / 2=(l+1) / 2$ the relation reads

$$
-2 p_{l-1}\left(\alpha_{l-1, m}-1 / 2\right)=2(d-l+2) r_{l-2}\left(a_{l-1, m}-1 / 2\right)<0,
$$

but at $\alpha_{l-3, m}=l / 2$ we get

$$
l r_{l}\left(\alpha_{l-1, m}-1\right)=2(d-l+2) r_{l}\left(\alpha_{l-1, m}-1\right)>0,
$$

because $r_{l}$ is positive to the left of its smallest zero, which is greater than $\alpha_{l-1, m}$. In other words, $r_{l-2}$ is positive at $\alpha_{l-3, m}$, and negative at $\alpha_{l-1, m}$ and then changes the sign between the subsequent zeros of $p_{l-1}$ because $r_{l}$ does so. At the last but one zero (of $p_{l-1}$ ) it thus has the sign of $(-1)^{(d-l-1) / 2}$ (number of zeroes of $p_{l-1}$ except the last), and close to the left of the last zero $p_{l-1}$ and $r_{l}$ both have the sign $(-1)^{(d-l-1) / 2}$ because their leading terms are $(-\alpha)^{(d-l+1) / 2}$. However, they enter the relation (A24) with negative coefficients $(l+1-2 \alpha<0$ when $\alpha>d / 2)$ which means that $r_{l-2}$ changes sign additionally before the last root of $(d+1) / 2$. Last thing to check is the $d=0$ case, for which $r_{-1}=1-2 \alpha$. This finishes the proof that $r_{-1}$ has $1+d / 2$ real simple roots.

[1] A. Bermudez, M. A. Martin-Delgado, E. Solano, Phys. Rev. A 76, 041801 (R) (2007).

[2] Acosta-Humánez P. B., PhD Thesis, Universitat Politècnica de Catalunya (2009). arXiv:0906.3532

[3] Cooper F., Khare A. and Sukhatme U., Phys. Repts. 251 267-385 (1995).

[4] Acosta-Humánez P. B. and Blázquez-Sanz D., Discrete Contin. Dynam. Syst. Series B 10, 2\&3, 265-293 (2008).

[5] Acosta-Humánez P. B., Morales-Ruiz J. and Weil J. A., Rep. Math. Phys. 67(3), 305-374, (2011).

[6] Stachowiak T., Szydłowski M. and Maciejewski A. J., J. Math. Phys. 47032502 (2006).

[7] Stachowiak T., J. Math. Phys. 52, 012301 (2011).

[8] Kaplansky I., "An Introduction to differential algebra", Hermann, Paris (1957).

[9] Kovacic J., J. Symbolic Comput. 2(1): 3-34 (1986).

[10] Duval A. and Loday-Richaud M., Appl. Algebra Engrg. Comm. Comput. 3(3), 211-246, (1992).

[11] Juan J. Morales-Ruiz, "Differential Galois theory and non-integrability of Hamiltonian systems", (Birkhäuser Verlag, Basel, 1999). 